\title{
Active Iron in Tomato Plants as Affected by Potassium to Calcium Ratio Applied to the Soil
}

\author{
M. E. Husein \\ Soil Dept., Faculty of Agriculture, Cairo Univ., Egypt.
}

\begin{abstract}
7 THE SUITABILITY of five chemical extractants (EDTA, DTPA, O-
$\mathrm{Ph}, 2,2$ 'Bip and $\mathrm{HCl}$ ) to estimate the amount of active iron, is studied in greenhouse experiments, using tomato plants. Different levels of Fe (0, 5 and $10 \mathrm{ppm})$ in combination with various ratios of $\mathrm{K}$ : Ca fertilization (1:20, 1:10 and 1:5) were used. The shoots of the harvested plants after 60 and 75 days from planting were analyzed for their total Fe, K, Ca, P and active Fe content as extracted by the abovementioned extractants.
\end{abstract}

The highest dry matter yield of tomato shoots at the two stages of growth were associated with $0 \mathrm{ppm} \mathrm{Fe}$ and 1:20 (K: Ca) ratio treatment and the least yield was recorded at the case of $0 \mathrm{ppm} \mathrm{Fe}$ and 1:5 (K: Ca) ratio. Iron application significantly increased the $\mathrm{Ca}$ content of the plants but decreased both $\mathrm{K}$ and $\mathrm{P}$, while total iron showed an insignificant increase. Increasing $\mathrm{K}$ : $\mathrm{Ca}$ ratio increased $\mathrm{K}, \mathrm{P}$ and $\mathrm{Fe}$ but decreased $\mathrm{Ca}$ content.

Active iron extracted using the previous methods increased with iron application increment and decreased by increasing $\mathrm{K}$ : Ca ratio. The extractability power, of the five solutions has the following ascending order: EDTA $<$ DTPA $<\mathrm{O} . \mathrm{Ph}<2,2$ 'Bip $<\mathrm{HCl}$.

The data illustrate that deficiency is expected at active iron levels less than 58,67, 116, 146 and $221(\mathrm{mg} / \mathrm{kg})$, for the 60-days old tomato shoots, and 49, 56, 63, 84 and $105(\mathrm{mg} / \mathrm{kg})$ for the 75 days old tomato shoots in the same order.

Keywords: Active iron, Chemical extractants, Tomato plants.

Iron is an essential element for plant growth and iron-deficiency induced chlorosis is a widespread nutritional problem. This disorder becomes evident as a typical yellowing of young leaves of plants in many crops and affects leaf and flower mineral composition and is responsible for significant decreases in yield, crop size and the quality of many species (Tagliavini and Rambola, 2001).

Plant analysis is one of the accepted tools for diagnosing deficiency disorders. Deficient plants, if analyzed at the right stage usually contain lower amount of the deficient element than the corresponding healthy checks. Perhaps, iron is the only essential element which often evades this most simple definition of deficiency, 
since instance is not uncommon when the total content of iron in the chlorotic plants was higher than in the green plants (Mehrotra et al., 1990).

Several techniques based on plant tissue analysis have been proposed for diagnosis of iron deficiency in plants (Katyal and Sharma, 1980 and Mehrotra et $a l, 1985)$. Various extractants have been proposed to extract the fraction of total iron, which is metabolically active and is related to occurrence of iron chlorosis. These extractants include water, dilute acids (hydrochloric acid, acetic acid, oxalic acid and citric acid), chelating agents such as EDTA, DTPA, tartaric acid and some organic solvents including 22'Bipyridyi and its derivatives, o-phenanthroline and several other compounds.

Some recent studies showed that when the chlorosis symptoms occurred, $\mathrm{K}$ contents of the plant were found high at these chlorotic plant samples (Torres et al., 2006 and Çelik and Katkat (2007). Hence the iron status of a leaf is also governed by relative amounts of potassium and calcium (DeKock, 1981). Abadia et al. (1985) studied the effect of Fe chlorosis on the mineral composition of field grown Peach tree leaves in two different areas in the Ebro valley. They found that potassium and $\mathrm{K} / \mathrm{Ca}$ ratio were significantly higher in chlorotic than in green or slightly chlorotic samples.

This study aims to identify the suitability of five chemical extractants to estimate the amount of iron, as an index of iron nutrition in the tissue of tomato plants grown under different levels of added $\mathrm{Fe}$ in combination with application of various ratios of $\mathrm{K}$ : $\mathrm{Ca}$.

\section{Materials and Methods}

Greenhouse experiment was carried out with tomato plant, as an indicator for active iron. Different levels of $\mathrm{Fe}$ in combination with various ratios of $\mathrm{K}$ : $\mathrm{Ca}$ fertilization were used. Five kilograms of pure fine sand were introduced into plastic pots. Ten seeds of tomatoes were uniformly sown per pot and brought daily to field moisture capacity by addition of Hoagland solution throughout the growth period. Two weeks after sowing, the plants were thinned to five plants per pot. At the same time both $\mathrm{K}$ : $\mathrm{Ca}$ and $\mathrm{CaCl}_{2}$ solutions having 1:20, 1:10 and 1:5 K: Ca ratios were mixed with Hoagland solution to each pot. Also, iron was sprayed to the plants at a rate of 0,5 or $10(\mathrm{mg} / \mathrm{kg})$ Fe-EDTA. The experiment comprised of nine treatments with 10 replicates of each.

After 60 days, top of the plants of five replicates of each treatment was harvested. Active iron in the fresh plant materials was determined using five specific reagents as follows:

- O-phenanthroline solution (O.Ph) (1.5\%, pH 3.0) according to the method described by Katyal and Sharma (1980 \& 1984).

- 2,2' Bipyridyl solution (2,2'Bip.) (1.5\%, $\mathrm{pH} 7.0$ ), according to the method described by Abadia (1984). 
- 0.1N ethylendiamine tetraacetic acid (EDTA), $\mathrm{pH} 7.0$, according to the method described by Mehrotra et al. (1985).

- $0.1 \underline{N}$ diethylen triamine pentaacetic acid (DTPA) $\mathrm{pH} 7.0$, according to the method described by Mehrotra et al. (1985).

$-0.1 \underline{N} \mathrm{HCl}$, according to the method described by Takkar and Kaur (1984).

The procedure involves extraction of $2 \mathrm{~g}$ of washed chopped fresh plant material by $20 \mathrm{ml}$ of each of the five reagents and $\mathrm{Fe}^{2+}$ was estimated directly in the filtrate by using atomic absorption spectrophotometer. (Unicam 969 AA Spectrometer, JERMANY) according to Page et al. (1982). The total iron and Ca were determined in plant digest using atomic absorption spectrophotometer. Potassium was determined in plant digest using flame photometer as described by Page et al. (1982). Phosphorus was determined in plant digest using ascorbic acid method described by Page et al. (1982).

The age of the extractant solution may play a role because 1,10-phenanthroline had lost part of its chelation capacity after 6 weeks. The ratio of leaf weight:extractant volume did not influence the amount of $\mathrm{Fe}$ extracted, provided the same amount of chelator was supplied (Zohlen, 2000).

\section{Results and Discussion}

Data in Table 1 and Fig.1 illustrate the response of dry matter yield of tomato shoots to different levels of iron and $(\mathrm{k}: \mathrm{Ca})$ ratio at the two stages of tomato plants growth.

Dry matter yield of tomato shoots increament after 60 and 75 days was 40 and $54 \%$, when the iron application was increased from 5 to $10(\mathrm{mg} / \mathrm{kg})$, respectively. For all iron treatments and at the two stages of growth, the dry matter formation of shoots gradually decreased as the $\mathrm{K}: \mathrm{Ca}$ ratio increased. The reduction in dry matter of shoots increased as the $\mathrm{K}: \mathrm{Ca}$ ratio increased. After 60 days from planting, the reduction in dry matter reached 20.37 and; $5.47 \%$ as an average due to the increase in $\mathrm{K}: \mathrm{Ca}$ ratio from $R_{1}$ (1:20) to $R_{2}(1: 10)$ and from $R_{2}(1: 10)$ to $R_{3}(1: 5)$, respectively, while after 75 days, the effect of increasing the K:Ca ratio was more pronounced resulting in a decrease percentage of 22.06 and 33.70 as the ratio increased for $R_{1}$ to $R_{2}$ and $R_{2}$ to $R_{3}$ , respectively. This clearly shows the adverse effect of increasing the K: Ca ratio on the dry matter production of tomato plants. Similar results were obtained by Guzmaz et al. (1986) and Therios et al.(2005).

Statistical analysis showed a highly significant effect of the interaction between added iron and $\mathrm{K}$ : Ca ratios on the dry matter of tomato plants at the two stages of growth (60 and 75 days). Such interaction was more pronounced with the application of $10 \mathrm{ppm} \mathrm{Fe}$ than the other two levels ( 0 and $5 \mathrm{ppm} \mathrm{Fe}$ ) at all ratios of $\mathrm{K} / \mathrm{Ca}$.

\section{Total iron content}

The total iron content of shoots after 60 and 75 days from planting is presented

Egypt. J. Soil Sci. 55, No. 4 (2015) 
in Table 2. The data showed that no marked differences were observed in the total iron contents between plants supplied or non with iron. It could be concluded that the total iron content does not always give a good indication of the iron status of the plant. Katyal and Sharma (1980 and 1984) indicated that total iron concentrations in plant are a poor indicator of iron status of plants. In several cases the deficient or chlorotic plants may contain similar or even more iron than the healthy ones.

Total leaf iron $(\mathrm{Fe})$ does not often correlate well with Fe deficiency symptoms. The extraction of 'active' Fe by $1 \mathrm{M} \mathrm{HCl}$ or Fe chelators, e.g., 1,10-phenanthroline, may reflect the relation between chlorosis and $\mathrm{Fe}$-concentration in the leaves better than total Fe does (Zohlen, 2000).

TABLE 1. Dry matter content (g/pot) of tomato plants.

\begin{tabular}{|c|c|c|c|c|}
\hline \multirow{2}{*}{$\begin{array}{c}\text { Iron } \\
\text { Treatment }\end{array}$} & \multicolumn{3}{|c|}{ K/Ca ratios } & \multirow{2}{*}{ Mean } \\
\hline & $\mathbf{R}_{1}$ & $\mathbf{R}_{1}$ & $\mathbf{R}_{1}$ & \\
\hline & \multicolumn{4}{|c|}{ First sample } \\
\hline $\mathrm{Fe}_{0}$ & 1.38 & 1.11 & 0.67 & 1.05 \\
\hline $\mathrm{Fe}_{1}$ & 2.00 & 1.64 & 1.57 & 1.73 \\
\hline $\mathrm{Fe}_{2}$ & 2.97 & 2.31 & 2.02 & 2.43 \\
\hline Mean & 2.11 & 1.68 & 1.42 & \\
\hline L.S.D & \multicolumn{2}{|c|}{$\begin{array}{c}\mathrm{Fe} \text { and } \mathrm{K} / \mathrm{Ca} \\
\text { ratio }\end{array}$} & \multicolumn{2}{|c|}{$\begin{array}{c}(\mathrm{Fe} \times \mathrm{K} / \mathrm{Ca} \\
\text { ratio })\end{array}$} \\
\hline$(.05)$ & \multicolumn{2}{|c|}{0.25} & \multicolumn{2}{|c|}{0.44} \\
\hline \multirow[t]{2}{*}{$(.01)$} & \multicolumn{2}{|c|}{0.35} & \multicolumn{2}{|c|}{0.61} \\
\hline & \multicolumn{4}{|c|}{ Second sample (leaves + stems) } \\
\hline $\mathrm{Fe}_{0}$ & 4.07 & 2.68 & 1.63 & 2.79 \\
\hline $\mathrm{Fe}_{1}$ & 5.36 & 4.25 & 2.57 & 4.06 \\
\hline $\mathrm{Fe}_{2}$ & 7.71 & 6.43 & 4.66 & 6.26 \\
\hline Mean & 5.71 & 4.45 & 2.95 & \\
\hline L.S.D & \multicolumn{2}{|c|}{$\begin{array}{c}\mathrm{Fe} \text { and } \mathrm{K} / \mathrm{Ca} \\
\text { ratio }\end{array}$} & \multicolumn{2}{|c|}{$\begin{array}{c}(\mathrm{Fe} \times \mathrm{K} / \mathrm{Ca} \\
\text { ratio })\end{array}$} \\
\hline$(.05)$ & \multicolumn{2}{|c|}{0.65} & \multicolumn{2}{|c|}{1.12} \\
\hline$(.01)$ & \multicolumn{2}{|c|}{0.89} & \multicolumn{2}{|c|}{1.55} \\
\hline $\begin{array}{l}\mathrm{Fe}_{0}: 0 \mathrm{ppm} \\
\mathrm{Fe}_{1}: 5 \mathrm{ppm} \\
\mathrm{Fe}_{2}: 10 \mathrm{ppm}\end{array}$ & & & $\begin{array}{l}: 0.05 \\
: 0.10 \\
: 0.20\end{array}$ & $\begin{array}{l}\text { Ca ratio } \\
\text { Ca ratio } \\
\text { Ca ratio }\end{array}$ \\
\hline
\end{tabular}

Egypt. J. Soil Sci. 55, No. 4 (2015) 

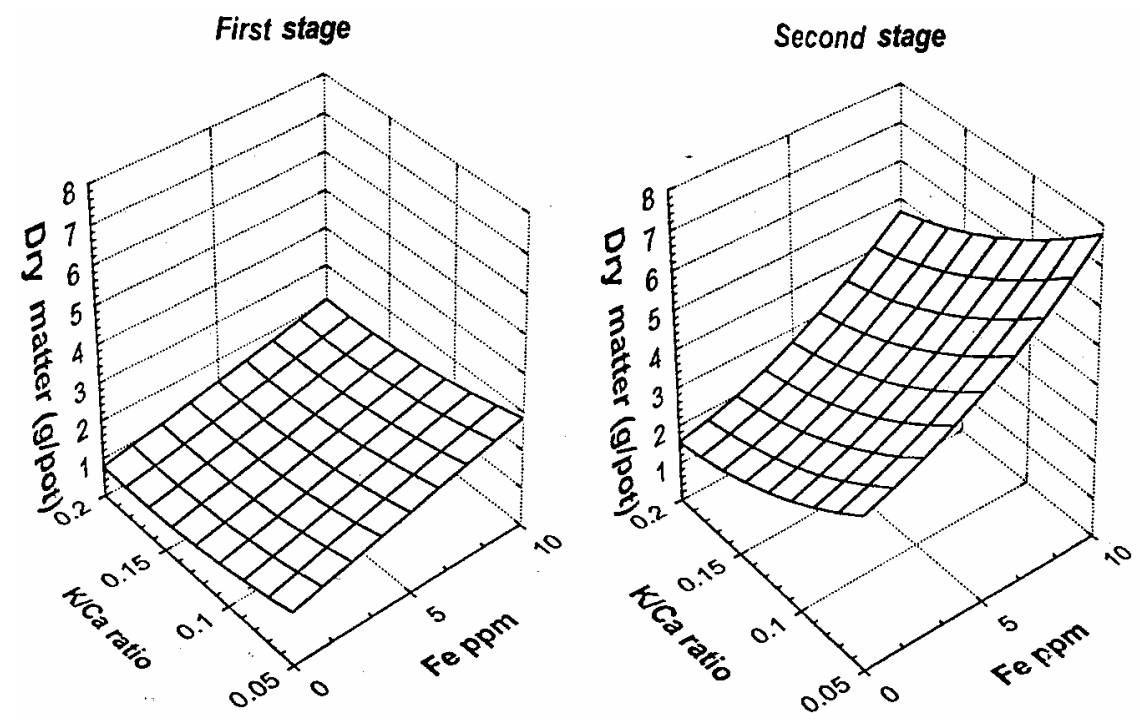

Fig. 1. Dry matter yield of tomato shoots grown under different levels of iron and $\mathrm{K} / \mathrm{Ca}$ ratio at two stage of growth.

TABLE 2. Total iron content $(\mathrm{mg} / \mathrm{kg})$ in tomato plants.

\begin{tabular}{|c|c|c|c|c|}
\hline \multirow{2}{*}{$\begin{array}{c}\text { Iron } \\
\text { treatments }\end{array}$} & \multicolumn{3}{|c|}{$\mathrm{K} / \mathrm{Ca}$ ratios } & \multirow[t]{2}{*}{ Mean } \\
\hline & $R_{1}$ & $\mathbf{R}_{2}$ & $\mathbf{R}_{\mathbf{3}}$ & \\
\hline & \multicolumn{4}{|c|}{ First sample } \\
\hline $\mathrm{Fe}_{0}$ & 465.00 & 486.33 & 499.00 & 483.44 \\
\hline $\mathrm{Fe}_{1}$ & 507.00 & 533.33 & 572.33 & 537.55 \\
\hline $\mathrm{Fe}_{2}$ & 533.67 & 570.67 & 585.00 & 563.11 \\
\hline Mean & 501.89 & 530.11 & 552.11 & \\
\hline L.S.D & \multicolumn{2}{|c|}{$\mathrm{Fe}$ and K/Ca ratio } & \multicolumn{2}{|c|}{ (Fe x K/Ca ratio) } \\
\hline$(.05)$ & \multicolumn{2}{|c|}{106.94} & \multicolumn{2}{|c|}{185.23} \\
\hline \multirow[t]{3}{*}{$(.01)$} & \multicolumn{2}{|c|}{147.35} & \multicolumn{2}{|c|}{255.21} \\
\hline & \multirow{2}{*}{\multicolumn{4}{|c|}{ Second sample }} \\
\hline & & & & \\
\hline $\mathrm{Fe}_{0}$ & 474.83 & 503.94 & 515.76 & 498.17 \\
\hline $\mathrm{Fe}_{1}$ & 505.58 & 516.58 & 525.50 & 515.88 \\
\hline $\mathrm{Fe}_{2}$ & 526.66 & 543.47 & 545.97 & 538.70 \\
\hline Mean & 502.35 & 521.33 & 529.07 & \\
\hline L.S.D & \multicolumn{2}{|c|}{ Fe and K/Ca ratio } & \multicolumn{2}{|c|}{ (Fe x K/Ca ratio) } \\
\hline$(.05)$ & \multicolumn{2}{|c|}{99.01} & \multicolumn{2}{|c|}{171.49} \\
\hline$(.01)$ & \multicolumn{2}{|c|}{136.42} & \multicolumn{2}{|c|}{236.29} \\
\hline
\end{tabular}

Moreover, the increase in the $\mathrm{K}$ : $\mathrm{Ca}$ ratio brought about slight but Egypt. J. Soil Sci. 55, No. 4 (2015) 
insignificant increases in the total iron concentration.

\section{Total potassium content}

Data of total potassium contents in plant tissues are given in Table 3. The obtained results show that potassium concentration ranged between 1.78 and $4.24 \%$ in the 60 days old shoots. Corresponding values for the shoots at 75 days old plants were $1.64-3.30 \%$. Application of $\mathrm{Fe}$ at the two levels of 5 and $10(\mathrm{mg} / \mathrm{kg})$ lead to gradual decrease in $\mathrm{K}$ content of shoots under different ratios of $\mathrm{K}: \mathrm{Ca}$ at the two stages of growth. Similar results were obtained by Allen et al. (2006). Potassium and iron are the main elements that are of great importance in soilless medium in terms of tomato yield and fruit quality. The interaction between potassium and iron applications was significant in yield. These results indicated interactive effects between potassium and iron applications on fruit quality and yield are more complex structure, (Filiz and Sahriye, 2010)

Moreover, total $\mathrm{K}$ contents of shoots significantly increased as the $\mathrm{K}$ : $\mathrm{Ca}$ ratio increased, under conditions of all iron treatments, and at the two stages of growth. Statistical analysis showed that the interaction between added iron and $\mathrm{K}$ : Ca ratios had highly significant effect on the potassium content in tomato plants at the two stages of growth (60 and 75 days).

TABLE 3. Total contents of potassium $(\%)$ in tomato plants.

\begin{tabular}{|c|c|c|c|c|}
\hline Iron & \multicolumn{3}{|c|}{ K/Ca ratios } & \multirow{2}{*}{ Mean } \\
\hline treatments & $\mathbf{R}_{1}$ & $\mathbf{R}_{2}$ & $\mathbf{R}_{3}$ & \\
\hline & \multicolumn{4}{|c|}{ First sample } \\
\hline $\mathrm{Fe}_{0}$ & 2.27 & 3.41 & 4.24 & 3.30 \\
\hline $\mathrm{Fe}_{1}$ & 2.07 & 3.07 & 3.94 & 3.02 \\
\hline $\mathrm{Fe}_{2}$ & 1.78 & 2.80 & 3.70 & 2.76 \\
\hline Mean & 2.04 & 3.09 & 3.96 & \\
\hline L.S.D & \multicolumn{2}{|c|}{$\mathrm{Fe}$ and $\mathrm{K} / \mathrm{Ca}$ ratio } & \multicolumn{2}{|c|}{$(\mathrm{Fe} \times \mathrm{K} / \mathrm{Ca}$} \\
\hline$(.05)$ & \multicolumn{2}{|c|}{0.26} & \multicolumn{2}{|c|}{0.44} \\
\hline \multirow[t]{2}{*}{$(.01)$} & \multicolumn{2}{|c|}{0.35} & \multicolumn{2}{|c|}{0.61} \\
\hline & \multicolumn{4}{|c|}{ Second sample } \\
\hline $\mathrm{Fe}_{0}$ & 1.83 & 2.72 & 3.30 & 2.61 \\
\hline $\mathrm{Fe}_{1}$ & 1.71 & 2.44 & 3.14 & 2.43 \\
\hline $\mathrm{Fe}_{2}$ & 1.64 & 2.25 & 2.92 & 2.27 \\
\hline Mean & 1.72 & 2.47 & 3.12 & \\
\hline L.S.D & \multicolumn{2}{|c|}{$\mathrm{Fe}$ and $\mathrm{K} / \mathrm{Ca}$ ratio } & \multicolumn{2}{|c|}{$(\mathrm{Fe} \times \mathrm{K} / \mathrm{Ca}$} \\
\hline$(.05)$ & \multicolumn{2}{|c|}{0.15} & \multicolumn{2}{|c|}{0.26} \\
\hline$(.01)$ & \multicolumn{2}{|c|}{0.21} & \multicolumn{2}{|c|}{0.36} \\
\hline $\begin{array}{l}\mathrm{Fe}_{0}: 0 \mathrm{ppm} \mathrm{Fe} \\
\mathrm{Fe}_{1}: 5 \mathrm{ppm} \mathrm{Fe} \\
\mathrm{Fe}_{2}: 10 \mathrm{ppm} \mathrm{Fe}\end{array}$ & & $\begin{array}{l}0.05 \\
0.10 \\
0.20\end{array}$ & $\begin{array}{l}\text { a ratic } \\
\text { a rati } \\
\text { a ratic }\end{array}$ & \\
\hline
\end{tabular}

Egypt. J. Soil Sci. 55, No. 4 (2015) 


\section{Total calcium content}

Table 4 shows that, as the level of iron application increased the calcium concentration in plants. Application of $\mathrm{Fe}$ at the two levels of 5 and $10 \mathrm{ppm}$ increased the $\mathrm{Ca}$ content of shoots under different ratios $\mathrm{K}: \mathrm{Ca}$ at the two stages of growth. Moreover, as the $\mathrm{K}: \mathrm{Ca}$ ratio increased significant decreases in $\mathrm{Ca}$ concentration were obtained. Similar conclusion was recorded by Cook et al. (1991)

\section{TABLE 4. Total contents of calcium (\%) in tomato plants.}

\begin{tabular}{|c|c|c|c|c|}
\hline Iron & \multicolumn{3}{|c|}{ K/Ca ratios } & \multirow[b]{2}{*}{ Mean } \\
\hline treatments & $\overline{\mathbf{R}_{1}}$ & $\mathbf{R}_{2}$ & $\mathbf{R}_{\mathbf{3}}$ & \\
\hline & \multicolumn{4}{|c|}{ First sample } \\
\hline $\mathrm{Fe}_{0}$ & 2.10 & 1.67 & 1.32 & 1.69 \\
\hline $\mathrm{Fe}_{1}$ & 2.41 & 1.91 & 1.62 & 1.98 \\
\hline $\mathrm{Fe}_{2}$ & 2.50 & 2.10 & 1.98 & 2.19 \\
\hline Mean & 2.33 & 1.89 & 1.64 & \\
\hline L.S.D & \multicolumn{2}{|c|}{$\mathrm{Fe}$ and $\mathrm{K} / \mathrm{Ca}$ ratio } & \multicolumn{2}{|c|}{ (Fe $\times$ K/Ca ratio) } \\
\hline$(.05)$ & \multicolumn{2}{|c|}{0.20} & \multicolumn{2}{|c|}{0.35} \\
\hline \multirow[t]{2}{*}{$(.01)$} & \multicolumn{2}{|c|}{0.31} & \multicolumn{2}{|c|}{0.56} \\
\hline & \multicolumn{4}{|c|}{ Second sample } \\
\hline $\mathrm{Fe}_{0}$ & 1.90 & 1.50 & 1.01 & 1.48 \\
\hline $\mathrm{Fe}_{1}$ & 2.10 & 1.76 & 1.10 & 1.68 \\
\hline $\mathrm{Fe}_{2}$ & 2.20 & 1.89 & 1.58 & 1.89 \\
\hline Mean & 2.06 & 1.71 & 1.23 & \\
\hline L.S.D & \multicolumn{2}{|c|}{$\mathrm{Fe}$ and $\mathrm{K} / \mathrm{Ca}$ ratio } & \multicolumn{2}{|c|}{ (Fe $\times$ K/Ca ratio) } \\
\hline$(.05)$ & \multicolumn{2}{|c|}{0.19} & \multicolumn{2}{|c|}{0.32} \\
\hline$(.01)$ & \multicolumn{2}{|c|}{0.25} & \multicolumn{2}{|c|}{0.41} \\
\hline $\begin{aligned} \mathrm{Fe}_{0}: 0 \mathrm{ppm} \mathrm{Fe} \\
\mathrm{Fe}_{1}: 5 \mathrm{ppm} \mathrm{Fe} \\
\mathrm{Fe}_{2}: 10 \mathrm{ppm} \mathrm{Fe}\end{aligned}$ & \multicolumn{4}{|c|}{$\begin{array}{ll}\mathrm{R}_{1}: 0.05 \mathrm{~K} / \text { Ca ratio } \\
\mathrm{R}_{2}: 0.10 \mathrm{~K} / \text { Ca ratio } \\
\mathrm{R}_{3}: 0.20 \mathrm{~K} / \text { Ca ratio }\end{array}$} \\
\hline
\end{tabular}

Statistical analysis of the data showed that the interaction between added iron and $\mathrm{K}: \mathrm{Ca}$ ratios on the calcium concentration in tomato plants at the two stages of growth (60 and 75 days) was highly significant.

Egypt. J. Soil Sci. 55, No. 4 (2015) 


\section{Total phosphorus content}

Results of phosphorus concentration in plant tissue are given in Table 5. Iron application significantly decreased phosphorus concentration. Phosphorus content in shoots was higher in treatment received no iron than those supplied with iron. The increase of $\mathrm{K}$ : Ca ratio was associated with a slight increase in $\mathrm{P}$ concentration.

TABLE 5. Total contents of phosphorus (\%) in tomato plants.

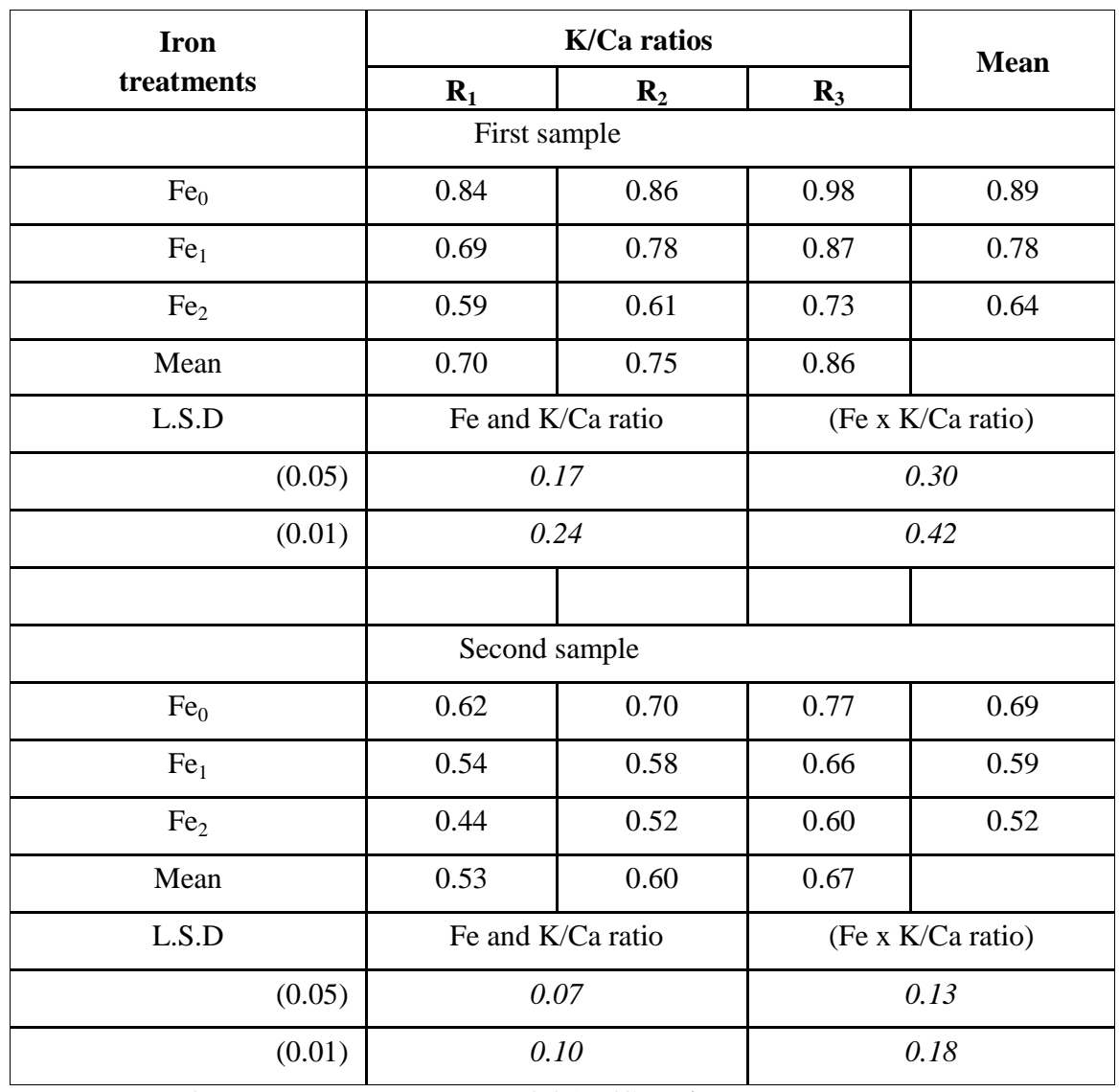

$\mathrm{Fe}_{0}: 0 \mathrm{ppm} \mathrm{Fe}$

$\mathrm{R}_{1}: 0.05 \mathrm{~K} / \mathrm{Ca}$ ratio

$\mathrm{Fe}_{1}: 5 \mathrm{ppm} \mathrm{Fe} \quad \mathrm{R}_{2}: 0.10 \mathrm{~K} / \mathrm{Ca}$ ratio

$\mathrm{Fe}_{2}: 10 \mathrm{ppm} \mathrm{Fe} \quad \mathrm{R}_{3}: 0.20 \mathrm{~K} / \mathrm{Ca}$ ratio

Active iron concentration:

Results recorderd in Table 6 show that the active iron extracted from the shoots of the two sampling dates increased by iron application and decreased by increasing the $\mathrm{K}$ :Ca ratio (Fig. 2- 6). 
TABLE 6. Active iron concentration $(\mathrm{mg} / \mathrm{kg})$ extracted by different methods from tomato plants.

\begin{tabular}{|c|c|c|c|c|c|c|c|c|}
\hline \multirow{3}{*}{$\begin{array}{c}\text { Iron } \\
\text { treatments }\end{array}$} & \multicolumn{4}{|c|}{ First samples } & \multicolumn{4}{|c|}{ Second samples } \\
\hline & \multicolumn{3}{|c|}{$\mathrm{K} / \mathrm{Ca}$ ratios } & \multirow[t]{2}{*}{ Mean } & \multicolumn{3}{|c|}{$\mathrm{K} / \mathrm{Ca}$ ratios } & \multirow[t]{2}{*}{ Mean } \\
\hline & $\mathbf{R}_{1}$ & $\mathbf{R}_{\mathbf{2}}$ & $\mathbf{R}_{\mathbf{3}}$ & & $\mathbf{R}_{1}$ & $\mathbf{R}_{\mathbf{2}}$ & $\mathbf{R}_{\mathbf{3}}$ & \\
\hline & \multicolumn{8}{|c|}{ EDTA } \\
\hline $\mathrm{Fe}_{0}$ & 74.17 & 59.93 & 45.15 & 59.75 & 63.51 & 54.25 & 40.48 & 52.74 \\
\hline $\mathrm{Fe}_{1}$ & 95.54 & 70.81 & 58.19 & 74.84 & 71.54 & 62.11 & 48.28 & 60.64 \\
\hline $\mathrm{Fe}_{2}$ & 124.57 & 100.00 & 84.56 & 103.04 & 82.70 & 72.04 & 56.67 & 70.47 \\
\hline Mean & 98.09 & 76.91 & 62.63 & & 72.58 & 62.80 & 48.47 & \\
\hline L.S.D & \multicolumn{2}{|c|}{$\mathrm{Fe}$ and $\mathrm{K} / \mathrm{Ca}$ ratio } & \multicolumn{2}{|c|}{ (Fe X K/Ca ratio) } & \multicolumn{2}{|c|}{$\mathrm{Fe}$ and $\mathrm{K} / \mathrm{Ca}$ ratio } & \multicolumn{2}{|c|}{ (Fe X K/Ca ratio) } \\
\hline$(.05)$ & \multicolumn{2}{|c|}{11.10} & \multicolumn{2}{|c|}{19.23} & \multicolumn{2}{|c|}{4.39} & \multicolumn{2}{|c|}{7.62} \\
\hline \multirow[t]{2}{*}{$(.01)$} & \multicolumn{2}{|c|}{15.30} & \multicolumn{2}{|c|}{26.50} & \multicolumn{2}{|c|}{6.06} & \multicolumn{2}{|c|}{10.49} \\
\hline & \multicolumn{8}{|c|}{ DTPA } \\
\hline $\mathrm{Fe}_{0}$ & 88.15 & 70.66 & 52.94 & 70.58 & 72.05 & 58.72 & 44.96 & 58.57 \\
\hline $\mathrm{Fe}_{1}$ & 98.84 & 83.04 & 62.80 & 81.56 & 83.16 & 68.68 & 55.92 & 69.25 \\
\hline $\mathrm{Fe}_{2}$ & 130.00 & 112.45 & 93.29 & 111.91 & 99.52 & 88.08 & 62.63 & 83.41 \\
\hline Mean & 105.66 & 88.71 & 69.66 & & 84.91 & 71.82 & 54.50 & \\
\hline L.S.D & $\mathrm{Fe}$ and & $\mathrm{K} / \mathrm{Ca}$ & (Fe $\mathrm{X}$ & $\mathrm{K} / \mathrm{Ca}$ & $\mathrm{Fe}$ and & $\mathrm{K} / \mathrm{Ca}$ & (Fe X & $\mathrm{K} / \mathrm{Ca}$ \\
\hline$(.05)$ & & & & 14 & & & & \\
\hline$(.01)$ & 12 & & & 23 & & & & \\
\hline
\end{tabular}

Egypt. J. Soil Sci. 55, No. 4 (2015) 
TABLE 6. Cont.

\begin{tabular}{|c|c|c|c|c|c|c|c|c|}
\hline \multirow{3}{*}{$\frac{\text { Iron }}{\text { treatment }}$} & \multicolumn{4}{|c|}{ First samples } & \multicolumn{4}{|c|}{ Second samples } \\
\hline & \multicolumn{3}{|c|}{$\mathrm{K} / \mathrm{Ca}$ ratios } & \multirow[t]{2}{*}{ Mean } & \multicolumn{3}{|c|}{$\mathrm{K} / \mathrm{Ca}$ ratios } & \multirow[t]{2}{*}{ Mean } \\
\hline & $\mathbf{R}_{1}$ & $\mathbf{R}_{2}$ & $\mathbf{R}_{\mathbf{3}}$ & & $\mathbf{R}_{1}$ & $\mathbf{R}_{2}$ & $\mathbf{R}_{\mathbf{3}}$ & \\
\hline & \multicolumn{8}{|c|}{ O-Ph. } \\
\hline $\mathrm{Fe}_{0}$ & $\begin{array}{c}103.0 \\
3\end{array}$ & 74.10 & 69.44 & 82.19 & 82.83 & 77.77 & 65.86 & 75.48 \\
\hline $\mathrm{Fe}_{1}$ & $\begin{array}{c}123.0 \\
1\end{array}$ & $\begin{array}{c}100.0 \\
0\end{array}$ & 91.70 & $\begin{array}{c}104.9 \\
0\end{array}$ & $\begin{array}{c}104.1 \\
7\end{array}$ & 86.83 & 72.39 & 87.79 \\
\hline $\mathrm{Fe}_{2}$ & $\begin{array}{c}159.5 \\
5\end{array}$ & $\begin{array}{c}132.1 \\
0\end{array}$ & $\begin{array}{c}111.0 \\
0\end{array}$ & $\begin{array}{c}134.2 \\
1\end{array}$ & $\begin{array}{c}125.5 \\
1\end{array}$ & $\begin{array}{c}109.4 \\
4\end{array}$ & 83.82 & $\begin{array}{c}106.2 \\
5\end{array}$ \\
\hline Mean & $\begin{array}{c}128.5 \\
3\end{array}$ & $\begin{array}{c}102.0 \\
6\end{array}$ & 90.71 & & $\begin{array}{c}104.1 \\
7\end{array}$ & 91.34 & 74.02 & \\
\hline L.S.D & \multicolumn{4}{|c|}{$\mathrm{Fe}$ and $\mathrm{K} / \mathrm{Ca}$ ratio $(\mathrm{Fe} \times \mathrm{K} / \mathrm{Ca}$} & \multicolumn{4}{|c|}{ Fe and $\mathrm{K} / \mathrm{Ca}$ ratio $(\mathrm{Fe} \times \mathrm{K} / \mathrm{Ca}$} \\
\hline$(.05)$ & \multicolumn{2}{|c|}{10.93} & \multicolumn{2}{|c|}{18.64} & \multicolumn{2}{|c|}{4.57} & \multicolumn{2}{|c|}{7.91} \\
\hline \multirow[t]{2}{*}{$(.01)$} & \multicolumn{2}{|c|}{14.82} & \multicolumn{2}{|c|}{25.68} & \multicolumn{2}{|c|}{6.29} & \multicolumn{2}{|c|}{10.91} \\
\hline & \multicolumn{8}{|c|}{ 2,2'Bip. } \\
\hline $\mathrm{Fe}_{0}$ & $\begin{array}{c}132.7 \\
3\end{array}$ & $\begin{array}{c}104.7 \\
0\end{array}$ & 82.44 & $\begin{array}{c}106.6 \\
2\end{array}$ & 99.53 & 91.20 & 72.06 & 87.59 \\
\hline $\mathrm{Fe}_{1}$ & $\begin{array}{c}158.5 \\
0\end{array}$ & $\begin{array}{c}127.9 \\
4\end{array}$ & $\begin{array}{c}108.6 \\
8\end{array}$ & $\begin{array}{c}131.7 \\
0\end{array}$ & $\begin{array}{c}124.1 \\
1\end{array}$ & $\begin{array}{c}105.3 \\
3\end{array}$ & 85.16 & $\begin{array}{c}105.8 \\
6\end{array}$ \\
\hline $\mathrm{Fe}_{2}$ & $\begin{array}{c}192.0 \\
5\end{array}$ & $\begin{array}{c}161.8 \\
4\end{array}$ & $\begin{array}{c}137.0 \\
3\end{array}$ & $\begin{array}{c}163.6 \\
4\end{array}$ & $\begin{array}{c}147.8 \\
5\end{array}$ & $\begin{array}{c}131.1 \\
6\end{array}$ & 95.19 & $\begin{array}{c}124.7 \\
3\end{array}$ \\
\hline Mean & $\begin{array}{c}161.0 \\
9\end{array}$ & $\begin{array}{c}131.4 \\
9\end{array}$ & $\begin{array}{c}109.3 \\
8\end{array}$ & & $\begin{array}{c}124.8 \\
3\end{array}$ & $\begin{array}{c}109.2 \\
3\end{array}$ & 84.13 & \\
\hline L.S.D & \multicolumn{4}{|c|}{$\begin{array}{r}\mathrm{Fe} \text { and } \mathrm{K} / \mathrm{Ca} \text { ratio } \\
\text { ratio })\end{array} \quad(\mathrm{Fe} \times \mathrm{K} / \mathrm{Ca}$} & \multicolumn{4}{|c|}{$\begin{array}{rr}\mathrm{Fe} \text { and } \mathrm{K} / \mathrm{Ca} \text { ratio } \\
\text { ratio })\end{array}\left(\begin{array}{l}(\mathrm{Fe} \times \mathrm{K} / \mathrm{Ca} \\
\end{array}\right.$} \\
\hline$(.05)$ & \multicolumn{2}{|c|}{21.58} & \multicolumn{2}{|c|}{40.85} & \multicolumn{2}{|c|}{5.43} & \multicolumn{2}{|c|}{9.41} \\
\hline \multirow[t]{2}{*}{$(.01)$} & \multicolumn{2}{|c|}{30.50} & \multicolumn{2}{|c|}{56.29} & \multicolumn{2}{|c|}{7.49} & \multicolumn{2}{|c|}{12.97} \\
\hline & & & & & & & & \\
\hline $\mathrm{Fe}_{0}$ & $\begin{array}{c}243.4 \\
9\end{array}$ & $\begin{array}{c}201.6 \\
0\end{array}$ & $\begin{array}{c}168.9 \\
0\end{array}$ & $\begin{array}{c}204.6 \\
6\end{array}$ & $\begin{array}{c}135.7 \\
6\end{array}$ & $\begin{array}{c}125.0 \\
3\end{array}$ & 88.45 & $\begin{array}{c}116.4 \\
1\end{array}$ \\
\hline $\mathrm{Fe}_{1}$ & $\begin{array}{c}277.2 \\
5\end{array}$ & $\begin{array}{c}231.1 \\
1\end{array}$ & $\begin{array}{c}215.7 \\
5\end{array}$ & $\begin{array}{c}241.3 \\
7\end{array}$ & $\begin{array}{c}0.7 \\
0\end{array}$ & $\begin{array}{c}137.7 \\
1\end{array}$ & $\begin{array}{c}118.5 \\
6\end{array}$ & $\begin{array}{c}138.9 \\
9\end{array}$ \\
\hline $\mathrm{Fe}_{2}$ & $\begin{array}{c}342.4 \\
7\end{array}$ & $\begin{array}{c}294.1 \\
8\end{array}$ & $\begin{array}{c}232.9 \\
0\end{array}$ & $\begin{array}{c}289.8 \\
5\end{array}$ & $\begin{array}{c}188.5 \\
4\end{array}$ & $\begin{array}{c}164.7 \\
7\end{array}$ & $\begin{array}{c}132.7 \\
9\end{array}$ & $\begin{array}{c}162.0 \\
3\end{array}$ \\
\hline Mean & $\begin{array}{c}287.7 \\
3\end{array}$ & $\begin{array}{c}242.2 \\
9\end{array}$ & $\begin{array}{c}205.8 \\
5\end{array}$ & & $\begin{array}{c}161.6 \\
6\end{array}$ & $\begin{array}{c}142.5 \\
0\end{array}$ & $\begin{array}{c}113.2 \\
6\end{array}$ & \\
\hline L.S.D & $\mathrm{Fe}$ and $\mathrm{H}$ & Ca ratio & $(\mathrm{Fe} x$ & Ca ratio & e and 1 & Ca ratio & $(\mathrm{Fe} x$ & JCa ratio \\
\hline$(.05)$ & & & & & & 54 & & \\
\hline$(.01)$ & & & & & & 80 & & 49 \\
\hline & $\begin{array}{l}\mathrm{Fe} \\
\mathrm{Fe} \\
\mathrm{Fe}\end{array}$ & $\begin{array}{r}0 \mathrm{ppm} \\
5 \mathrm{ppm} \\
10 \mathrm{ppm}\end{array}$ & & & & $\begin{array}{ll}R_{1} & : 0.0 \\
\hat{R}_{2} & : 0.1 \\
R_{3} & : 0.2\end{array}$ & $\begin{array}{l}\mathrm{K} / \mathrm{Ca} \mathrm{ra} \\
\mathrm{K} / \mathrm{Ca} \mathrm{ra} \\
\mathrm{K} / \mathrm{Ca} \mathrm{ra}\end{array}$ & \\
\hline
\end{tabular}

Egypt. J. Soil Sci. 55, No. 4 (2015) 

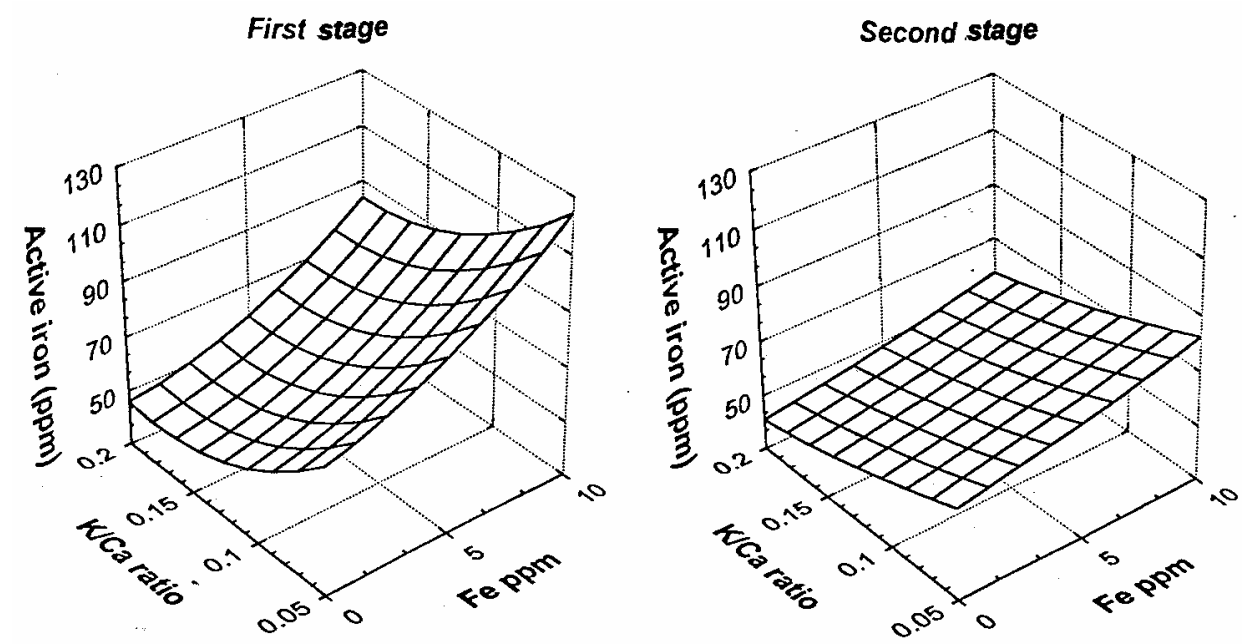

Fig. 2. Active Fe extracted by EDTA $(\mathrm{mg} / \mathrm{kg}$ ) of tomato shoots under different levels of iron and K/Ca at two stages of growth.
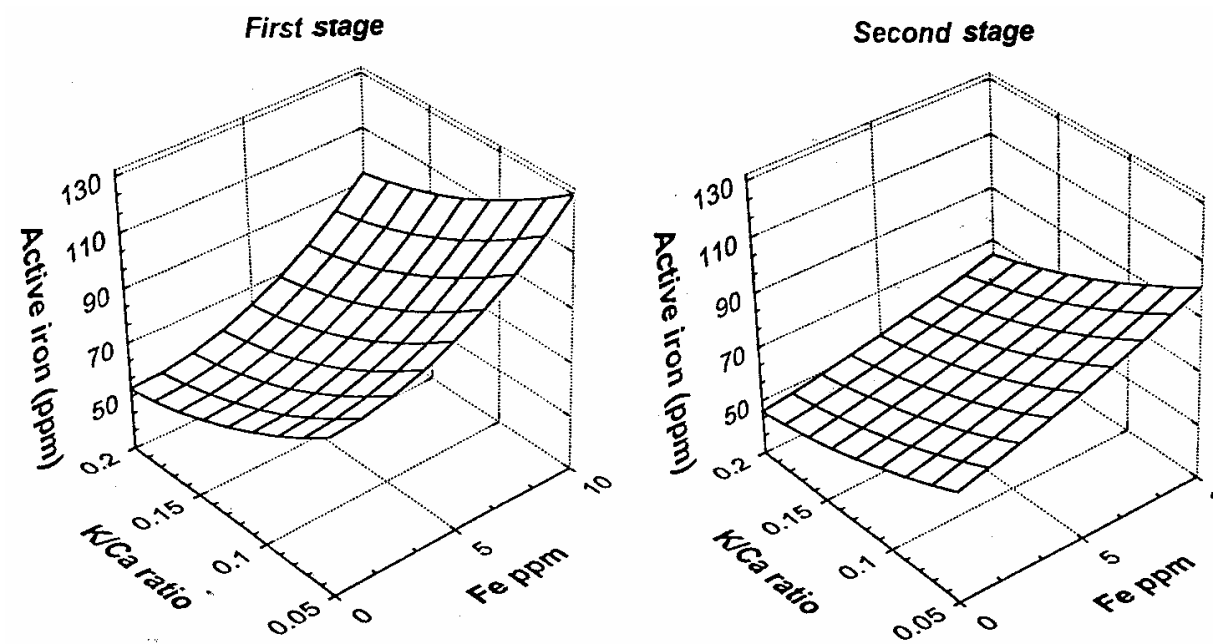

Fig. 3. Active Fe extracted by DTPA $(\mathrm{mg} / \mathrm{kg})$ of tomato shoots under different levels of iron and K/Ca at two stages of growth.

Egypt. J. Soil Sci. 55, No. 4 (2015) 

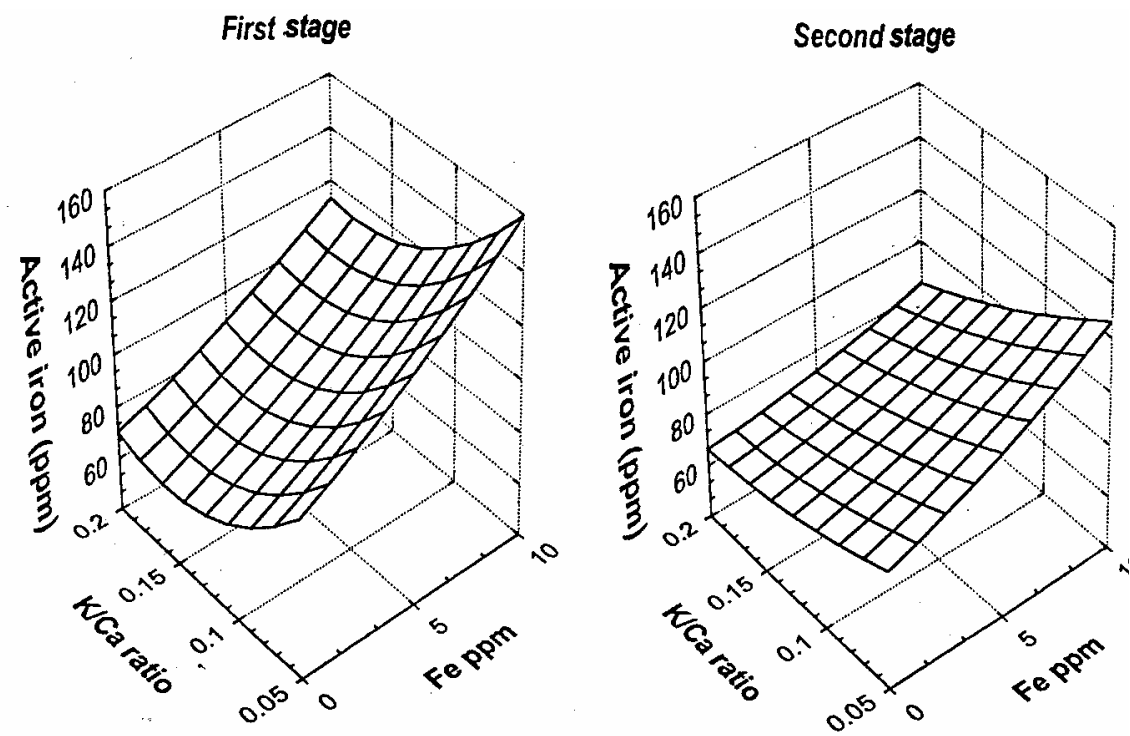

Fig. 4. Active Fe extracted by O-ph ( $\mathrm{mg} / \mathrm{kg})$ of tomato shoots under different levels of iron and K/Ca at two stages of growth.
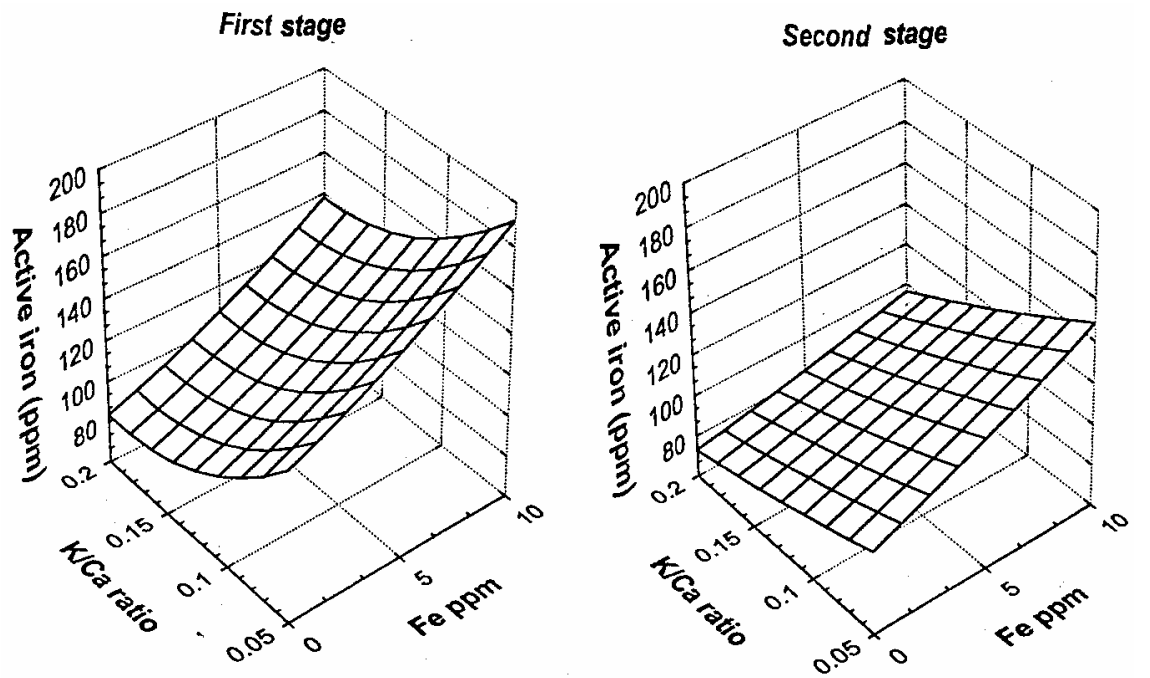

Fig.5. Active Fe extracted by 2,2 'Bip $(\mathrm{mg} / \mathrm{kg}$ ) of tomato shoots under different levels of iron and K/Ca at two stages of growth. 

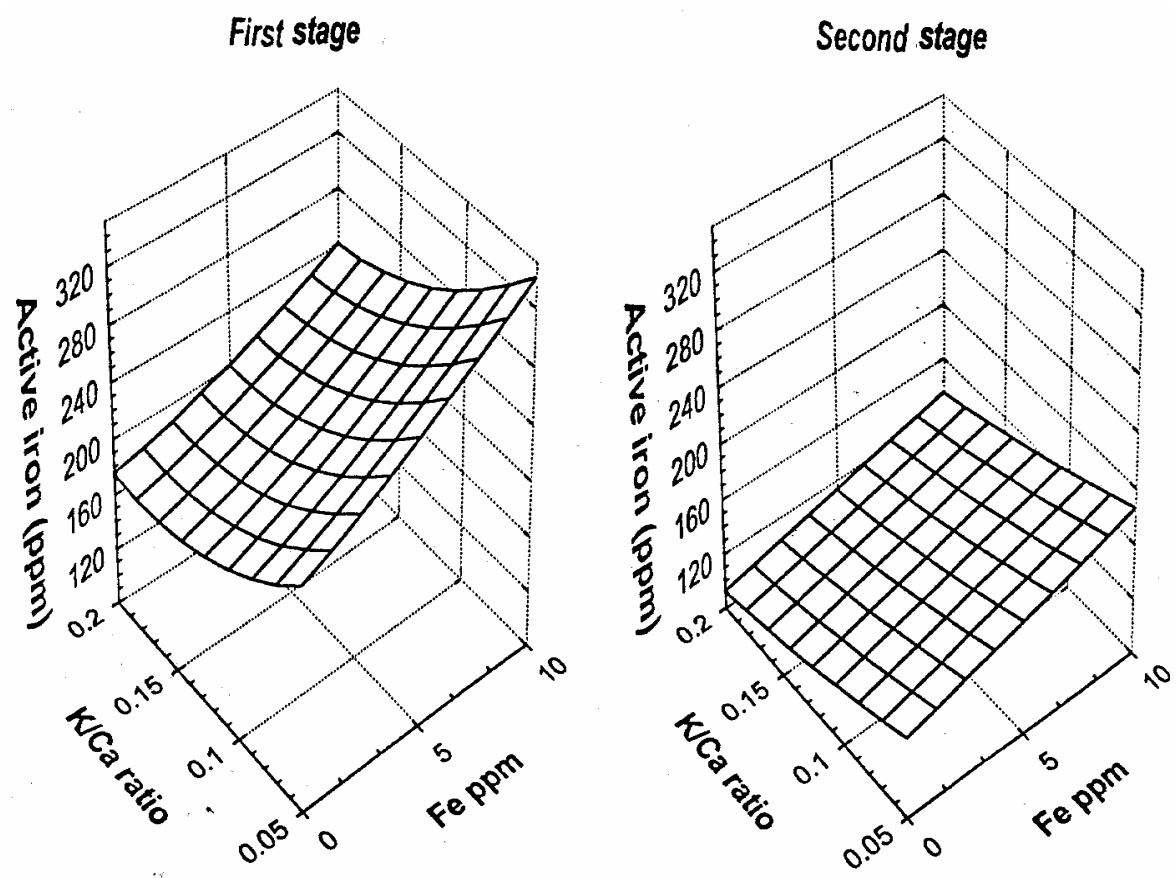

Fig. 6. Active Fe extracted by $\mathrm{HCl}(\mathrm{mg} / \mathrm{kg})$ of tomato shoots under different levels of iron and $\mathrm{K} / \mathrm{Ca}$ at two stages of growth

Statistical analysis showed that the interaction between added iron and K: $\mathrm{Ca}$ ratios had highly significant effect on the active iron in tomato plants at the two stages of growth (60 and 75 days). In general, the highest active iron content of shoots was associated with the treatment of $10 \mathrm{ppm} \mathrm{Fe}$ and 1:20 K: $\mathrm{Ca}$ ratio, while the lowest one was observed at 0 ppm Fe and 1:5 K: Ca ratio.

The correlation coefficients between active iron concentration and each of dry matter yield and total Fe content in the shoots of tomato plants at the two stages of growth are presented in Table 7. Significant correlations were obtained between dry matter yield of the first and second samples of shoots. However, no significant correlations were found between total iron content in the shoots at both stages of growth and dry matter yield. These results clearly demonstrate that none of the extractants examined in this study could be used for the assessment of the iron status in tomato plant. These findings agree with those reported by Mehrotra et al. (1985); Rao et al. (1987) and Aly (1994). Moreover, no significant correlations were obtained between the amount of $\mathrm{Fe}$ extracted from the shoots by any of the abovementioned extractants and total Fe content of the shoots at the two samples.

Egypt. J. Soil Sci. 55, No. 4 (2015) 
TABLE 7. Correlation coefficients between active iron extracted in different methods and each of dry matter yield and total iron contents in tomato plants at two stage of growth.

\begin{tabular}{|c|c|c|c|c|}
\hline \multirow{2}{*}{$\cdot$} & \multicolumn{4}{|c|}{ Correlation coefficient (r) } \\
\hline Extraction method & \multicolumn{2}{|c|}{ First sample } & \multicolumn{2}{c|}{ Second sample } \\
\cline { 2 - 5 } & $\begin{array}{c}\text { Dry } \\
\text { matter }\end{array}$ & $\begin{array}{c}\text { Total } \\
\text { Fe }\end{array}$ & $\begin{array}{c}\text { Dry } \\
\text { matter }\end{array}$ & $\begin{array}{c}\text { Total } \\
\text { Fe }\end{array}$ \\
\hline EDTA & $0.81^{* *}$ & $\mathrm{~ns}$ & $0.91^{* *}$ & $\mathrm{~ns}$ \\
\hline DTPA & $0.85^{* *}$ & $\mathrm{~ns}$ & $0.93^{* *}$ & $\mathrm{~ns}$ \\
\hline O-Ph. & $0.82^{* *}$ & $\mathrm{~ns}$ & $0.90^{* *}$ & $\mathrm{~ns}$ \\
\hline $2,2^{\prime} \mathrm{Bip}$. & $0.70^{* *}$ & $\mathrm{~ns}$ & $0.86^{* *}$ & $\mathrm{~ns}$ \\
\hline HCl & $0.63^{* *}$ & $\mathrm{~ns}$ & $0.76^{* *}$ & $\mathrm{~ns}$ \\
\hline Total Fe & $\mathrm{ns}$ & -- & $\mathrm{ns}$ & -- \\
\hline
\end{tabular}

ns : not significant

$*$ : significant at $5 \%$ probability.

** : significant at $1 \%$ probability.

Generally, the statistical analysis showed highly significant correlations between the values of active iron extracted by the five methods (EDTA, DTPA, o- $\mathrm{Ph}, 2,2$ 'Bip and $\mathrm{HCl}$ ) from tomato shoots after 60 or 75 days from planting (Table 8). Thus, each of these extractants could be effectively used to describe Fe status in the plants which grown in Egyptian soils.

TABLE 8. Correlation matrix between different methods used to extract active iron from tomato plant at two stages of growth.

\begin{tabular}{|c|c|c|c|c|}
\hline \multicolumn{5}{|c|}{ Extractants } \\
\hline \multirow{2}{*}{$\mathrm{HCl}$} & \multicolumn{1}{|c|}{ EDTA } & DTPA & $\begin{array}{c}\text { O- } \\
\text { Ph. }\end{array}$ & 2,2' Bip. \\
\cline { 2 - 5 } & \multicolumn{5}{|c|}{ First sample } \\
\hline & $0.57^{* *}$ & $0.38^{*}$ & $0.67^{* *}$ & $0.73^{* *}$ \\
\hline $2,2^{\prime}$ Bip. & $0.74^{* *}$ & $0.65^{* *}$ & $0.91^{* *}$ & \\
\hline O-Ph. & $0.88^{* *}$ & $0.72^{* *}$ & & \\
\hline DTPA & $0.57^{* *}$ & & & \\
\hline & & \multicolumn{5}{|c|}{ Second sample } \\
\hline HCl & $0.71^{* *}$ & $0.71^{* *}$ & $0.43^{*}$ & $0.65^{* *}$ \\
\hline $2,2^{\prime}$ Bip. & $0.90^{* *}$ & $0.89^{* *}$ & $0.55^{* *}$ & \\
\hline O-Ph. & $0.51^{* *}$ & $0.53^{* *}$ & & \\
\hline DTPA & $0.71^{* *}$ & & & \\
\hline
\end{tabular}

* : significant at $5 \%$ probability.

$* *$ : significant at $1 \%$ probability. 
The active Fe extracted by each of EDTA, DTPA, o-Ph, 2,2'Bip and $\mathrm{HCl}$ from tomato plants is calibrated against dry matter. Values of such critical level for the active iron extracted are shown in Table 9. As regards the yield response as a function of each of EDTA, DTPA, o-Ph, 2,2'Bip and $\mathrm{HCl}-$ extractable active iron, the data illustrate that deficiency is expected at active iron levels less than 57.76, 66.73, 116.30, 146.30, and $220.95 \mathrm{ppm}$, for the 60-days old shoots, respectively. Corresponding expected deficiency values for the 75-days old shoots are 48.79, 55.94, 63.37, 83.94 and 105.02 for the five extractants previously mentioned in the same order. In this respect, it could be observed that the critical level of active iron in the second sample for the five extractants is much lower than those in the first sample. This indicates that tomato plants require high amounts of $\mathrm{Fe}$ during their early stages of growth.

TABLE 9. Critical values of active iron (mg/kg) extracted by different extraction to clarify tomato plant iron status at the two stages of growth .

\begin{tabular}{|c|c|c|}
\hline \multirow{2}{*}{ Extraction } & \multicolumn{2}{|c|}{ Critical values } \\
\cline { 2 - 3 } & First sample & Second sample \\
\hline EDTA & 57.76 & 48.79 \\
\hline DTPA & 66.73 & 55.94 \\
\hline O-Ph. & 116.30 & 63.37 \\
\hline 2,2 ' Bip. & 146.30 & 83.94 \\
\hline HCl & 220.95 & 105.02 \\
\hline
\end{tabular}

\section{Conclusion}

Higher concentrations of active Fe were found in the non chlorotic leaves. These results are in accordance with other researchers (Karaman 1999 and Başar $2000 \& 2003)$. High concentrations of total iron in chlorotic leaves show that total iron is not a good indicator of the iron status of plants (Sönmez and Kaplan, 2004). This is evident from the negative correlation between active $\mathrm{Fe}$ and the degree of chlorosis ( Köseoğlu \& Açıkgöz, 1995 and Sönmez \& Kaplan, 2004). For evaluation of plant responses to various factors affecting $\mathrm{Fe}$ availability in the soil and $\mathrm{Fe}$ nutrition in plants, the concentration of active iron in leaves is recognized as a better nutritional iron indicator than total iron and has been also suggested by Mengel (1995).

\section{References}

Abadia, J., Monge, E. Montanes, L. and Heras, L. (1984) Extraction of iron from plant leaves by Fe(II) chelators. J. Plant Nutr. 7, 777 - 784.

Egypt. J. Soil Sci. 55, No. 4 (2015) 
Abadia, J., Nishio, J. N., Monge, E., Montanes, L. and Heras. L. (1985) Mineral composition of peach leaves affected by iron chlorosis. J. Plant Nutr. 8, 697 - 707.

Allen V. Barker and David J. Pilbeam. (2006) Handbook of Plant Nutrition. CRC Press, 632 pages

Aly, S. S. M. (1994) Effect of some chemical treatments on iron chlorosis recovery. Ph. D. Thesis, Fac of Agric. Ain Shams Univ.

Başar H. (2000) Factors affecting iron chlorosis observed in peach trees in the Bursa region. Turkish Journal of Agriculture and Forestry. - 2000, vol. 24, p. 237- 245.

Basar, H. (2003) Analytical methods for evaluating iron chlorosis in peach trees. Communications in Soil Science and Plant Analysis, 34, 327- 341.

Çelik, H. and Katkat, A. V. (2007) Some physical soil properties and potassium as an intensified factor on iron chlorosis. International Journal of Soil Science. - 2007, vol. 2 (4), p. 294- 300

Cook, J.D., Dassenko. S.A. and Whittaker P. (1991) Calcium supplementation: effect on iron absorption. Am J. Clin Nutr., 53(1), 106-11.

DeKock, P.C. (1981) Iron nutrition under conditions of stress. J. Plant Nutr., 3, 513 - 521.

Filiz, Ö. A. and Sahriye, S. (2010) Reflection of different application of potassium and iron fertilization on tomato yield and fruit quality in soilless medium. J. of Food, Agriculture and Environment, (8), 426-429.

Guzmaz, M., Urrestazaru, M. and Romero, L. (1986) Active and total iron in Castanea sativa and their relation to other nutrients. J. Plant Nutr. 9 (3-7), 909-921

Karaman, M.R. (1999) Investigation of chlorosis in peach trees growing in the Tokat region using soil and plant analysis and the effects of soil moisture on the determination of the amounts of DTPA-extractable $\mathrm{Fe}, \mathrm{Cu}, \mathrm{Zn}$ and Mn. Turk. J. Agric. For .23 (3), 707-719.

Katyal, J.C. and Sharma, B.D. (1980) A new technique of plant analysis to resolve iron chlorosis. Plant and Soil, 55, 105 - 119.

Katyal, J.C. and Sharma, B.D (1984) Some modification in the assay of $\mathrm{Fe}^{2+}$ in 1-10,o-phenanthroline extracts of fresh plant tissues. Plant and Soil, 79, 449 - 450.

Ko"seoglu, A. T. and Ac,iko"z. V. (1995) Determination of iron chlorosis with extractable iron analysis in peach leaves. J. of plant Nutrition, 18, 153-161

Mehrotra, S.C., Sharma, C.P. and Agarwala, S.C. (1985) A search for extractants to evaluate the iron status of plants. Soil Sci. Plant Nutr. 31, 155 - 162.

Mehrotra S.C, Gupta P, Chaturvedi K, and Bisht SS (1990) Active Fe in relation to chlorophyll and activities of some Fe-enzymes in maize. Indian Journal of Experimental Biology. 28, 349 - 351 
Mengel, K., (1995) Iron availability in plant tissue-iron chlorosis on calcareous soils. In Abadia J. (Ed) Iron Nutrition in Soils and Plants. Kluwer Academic, Dordrecht, The Netherlands, pp. 389-397.

Page, A.L., Miller, R. H. and Keeny, D. R. (1982) Methods of Soil Analysis Part 2. Chemical and Microbiological Properties $2^{\text {nd }}$ ed. Am. Soc. of Agron., Madison, Wisconsin, USA

Rao, J.K., Sahrawat, K.L. and Burford, J.R.(1987) Diagnosis of iron deficiency in groundnut, Arachis hypogaea L. Plant and Soil 97, 353 - 359.

So"nmez, S., and Kaplan, M.(2004) Comparison of various analysis methods for determination of iron chlorosis in apple trees. Journal of Plant Nutrition, 27, 2007- 2018.

Tagliavini M. and Rambola A.D. (2001) Iron deficiency and chlorosis in Orchard and Vineyard Ecosystems. European Journal of Agronomy, 15,71-92.

Takkar, P.N. and Kaur,N.P. (1984) $\mathrm{HCl}$ method for $\mathrm{Fe}^{2+}$ estimation to resolve iron chlorosis in plants. J. Plant Nutr. 7, $81-90$.

Therios I., Chouliaras, V., Bizas, D., Boukouvalas,S., Palioura,E.,Hatzidimitriou, E. Basioukas, D., Vassilios G. and Nikolaos S. (2005) Changes in Leaf Biochemical and Indices due to Iron Deficiency in Citrus. Agro Thesis Vo3, No. 1: 18-25

Torres, R. M., Barra, J. D. E., Gonzales G. A. et al., (2006) Morphological changes in leaves of Mexican lime affected by iron chlorosis. Journal of Plant Nutrition.2006, vol. 29, No. 4, p. 615- 28

Zohlen, A., (2000) Use of 1,10-phenanthroline in estimating metabolically active iron in plants. Communications in Soil Science and Plant Analysis, 31(3-4), pp.481 - 500.

(Received 21/1/2015;

accepted 4/10/2015) 


\section{الحديد النشط فى نباتات الطماطم ومدى تأثره بنسبة البوتاسيوم الى الكي \\ الكالسيوم المضاف الى التربة النية}

كلية الزراعة - جايني حسينة القاهرة - قسم الأراضى والمياه - مصر .

اجريت دراسة بهدف دراسة مدى ملائمة خمسة مستخلصات كيماوية التقدير كمية الحديد (EDTA, DTPA, O-Ph, 2,2'Bip and HCl) النشط كدليل على حالة الحديد في أنسجة نباتات الطماطم النامية في

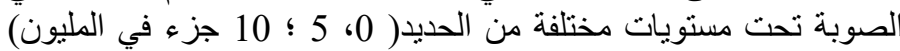
ونسب مختلفة من البوتاسيوم : الكالسيوم ( 1 : 20، 1 : 10، 1 : 1 : 5 ) )

وقد نم حصاد النباتات لكل معاملة بعد 60 ، 70 يوم من الزر اعة. وتــــ

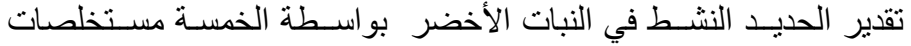

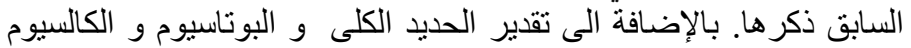
و الفوسفور في المادة الجافة للنبات الطماطم.

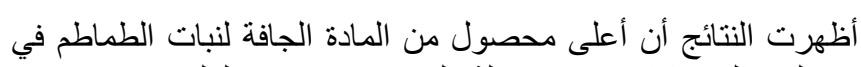

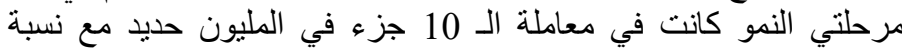

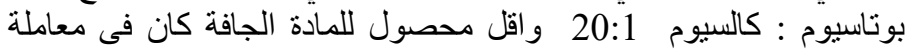

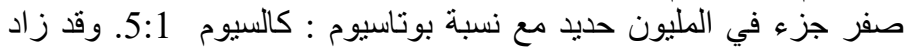

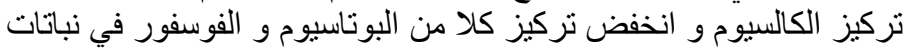

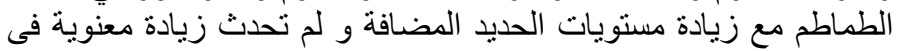

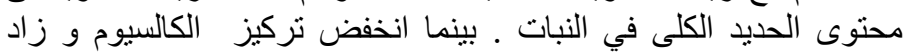
تركيز كلا من البوتاسيوم و الفوسفور و الحديد الكلى في نباتات الطئل الطماطم بزيادة نسبه البوتاسيوم : ألكالسيوم المضافة الطياط.

وقد أظهرت نتائج الحديد النشط المستخلص بواسطة المستخلصات

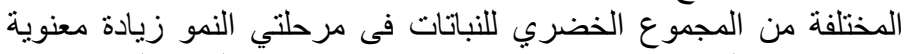

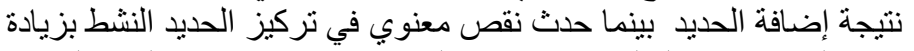

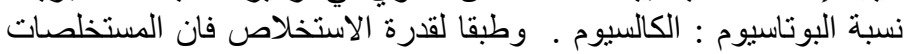
الخمسة المستعملة يمكن ترتيبها كما يلي:

EDTA < DTPA < O-Ph < 2,2'Bip. $<\mathrm{HCl}$

وتوضح النتائج المتحصل عليها أن المستوى الحرج التركيز الحديد

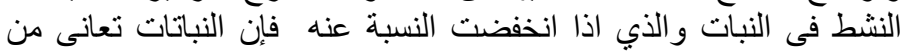

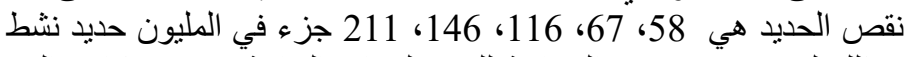

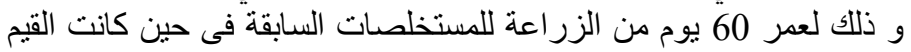

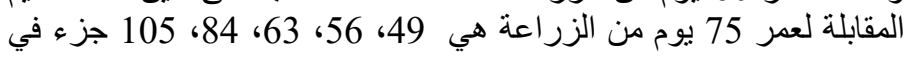
المليون حديد نتشط لنفس المستخلصات على التو الي. 